\title{
Estilos de crianza de madres con experiencias de violencia de pareja
}

\section{Parenting styles of mothers with partner violence experiences}

José Concepción Gaxiola-Romero

Rosa Melina Millanes-Vargas

Cynthia Lorenia Aranda-Corrales

Universidad de Sonora (UNISON)

\section{Resumen}

Las consecuencias de la violencia de pareja pueden expresarse en el tipo de crianza que las madres dan a sus hijos(as). El objetivo del presente estudio fue evaluar, en un contexto de violencia de pareja, los factores protectores de la crianza: las variables de apoyo social percibido de los amigos, familiares y de vecinos, bienestar psicológico, disposiciones a la resiliencia y la crianza autoritativa; además de factores de riesgo: las variables depresión, problemas de autorregulación y maltrato infantil. Previo consentimiento informado, fueron seleccionadas 254 madres con experiencia de violencia de pareja, a partir de sus respuestas a un cuestionario. La batería de pruebas construida según los objetivos del estudio evaluó características sociodemográficas y cada una de las variables seleccionadas. Se realizaron estadísticas descriptivas para las variables demográficas y se promediaron las respuestas de cada escala. Asimismo, fueron conformadas variables latentes para probar sus relaciones en un modelo hipotético de ecuaciones estructurales en el programa EQS 6.1. Los resultados mostraron dos caminos posibles: el primero, donde a partir de la violencia se encuentra la depresión y problemas de autorregulación que culminan en maltrato infantil; y segundo, donde a raíz de la violencia bajo la influencia del apoyo social percibido, el bienestar psicológico, la resiliencia, termina con la crianza autoritativa.

Palabras clave: madres, violencia de pareja, apoyo social, crianza autoritativa, maltrato infantil.

José Concepción Gaxiola Romero, Departamento de Psicología y Ciencias de la Comunicación, Universidad de Sonora (UNISON).

La correspondencia en relación con este artículo debe dirigirse a José Concepción Gaxiola Romero, Universidad de Sonora, UNISON. Blvd. Luis Encinas y Rosales S/N, colonia centro, C.P. 83000.

Dirección electrónica: joegaxiola@gmail.com 


\begin{abstract}
The consequences of intimate partner violence can be expressed in mother's childrearing practices with their own sons and daughters. The aim of this study was to evaluate factors of the upbringing of intimate partner violence, and the risk factors which are the variables depression, problems of self-regulation and child maltreatment and the protective factors being the variables of social support perceived by friends, family and neighbors, psychological well-being, dispositions to resilience and authoritative parenting style. With informant consent signed, 254 mothers with experience with intimate partner violence were selected from their answers given to a questionnaire. The battery of tests was constructed according to the objectives of the study, sociodemographic characteristics were evaluated and each one of the selected variables. Descriptive statistics were used to measure demographic variables, after that, the results of the scales were averaged to conform latent variables to test it in a hypothetical structural equation model in the EQS 6.1 program. The results showed two courses: one where from the violence is the depression and problems of self-regulation culminating in child abuse, and another where as a result of violence under the influence of perceived social support, psychological well-being, resilience to end authoritative parenting.
\end{abstract}

Keywords: mothers, intimate partner violence, social support, authoritative parenting style, child maltreatment.

La Organización Mundial de la Salud (OMS) define la violencia de pareja hacia la mujer como "todo acto de violencia de género que resulte, o pueda tener como resultado un daño, físico, sexual o psicológico para la mujer, inclusive las amenazas de tales actos, la coacción o la privación arbitraria de la libertad, tanto si se producen en la vida pública como privada" (2013a, p. 1). Asimismo, esta organización detectó (2013) una prevalencia mundial de 30\% de las mujeres que han sufrido violencia de pareja en algún momento de su vida. En México, 47 de cada 100 mujeres de 15 años y más, que han tenido al menos una relación de pareja, matrimonio o noviazgo, han sido agredidas (INEGI, 2016). En el estado de Sonora, el $52.1 \%$ de las mujeres casadas o en unión libre, alguna vez, han experimentado alguna situación de violencia por parte de sus parejas (Ibídem).

En los últimos años, se ha producido el aumento de las investigaciones sobre la violencia de pareja debido a su alta prevalencia, así como a sus graves consecuencias en la salud física y psicológica a corto y largo plazo (Alonso, Manso, García-Baamonde, \& Sánchez, 2009).

El apoyo social se identificó como factor importante en la relación violencia-depresión puesto que, al no percibir apoyo social, se presenta con mayor frecuencia la depresión en mujeres que viven violencia. De igual forma, percibir un mayor apoyo social disminuye la presencia de depresión en mujeres que hayan pasado por esta problemática de violencia, 
además, se presentó un impacto positivo en el bienestar psicológico (Buesa \& Calvete, 2013).

La crianza, en este caso, es una variable importante que difiere en distintos tipos de padres, es decir, existen diversos tipos. Según Darling y Steinberg (1993), destaca la crianza positiva (también conocida como autoritativa), que en este caso es la adecuada. Para Limiñana, Martínez y Pérez (2017), convivir con una pareja que agrede a la madre mientras los menores están presentes puede influir en su capacidad para instaurar normas y límites en sus hijos(as).

Puesto que las cifras de violencia de pareja van en aumento, el presente estudio busca demostrar las relaciones entre las variables mencionadas, que antes fueron estudiadas de manera aislada $\mathrm{y}$, en este caso, de manera conjunta y con base en la teoría del desarrollo de la psicopatología (Cicchetti, 2006), la cual establece que en el desarrollo humano se presentan múltiples combinaciones entre factores psicológicos, biológicos y sociales, cuyos resultados pueden ser adaptativos o desadaptativos; tal situación hace referencia al principio de multifinalidad, el cual explica que similares procesos pueden llevar a resultados diferentes. La teoría refiere que pueden existir variadas trayectorias en el progreso psicológico de las personas frente a experiencias con variables similares, incluso ante variables de riesgo.

En este sentido, desde un enfoque salutogénico y de adaptación psicológica, surge el interés de investigar las consecuencias de los factores de riesgo, pero también, el estudio de los posibles resultados positivos, con base en la influencia de factores protectores sobre los de riesgo (Jose \& Novaco, 2016); por lo tanto, se busca evidenciar los factores de riesgo y consecuencias negativas de la violencia de pareja y, al mismo tiempo, determinar si existen algunas variables que puedan proteger a las mujeres que la padecen, de sus efectos negativos, un planteamiento no considerado en conjunto en otras investigaciones. De este modo, se busca establecer un conocimiento para la formulación de estrategias de intervención, enfocadas en proteger a las madres de las consecuencias negativas de violencia de pareja.

\section{Factores de riesgo de la violencia de pareja hacia la mujer}

Con respecto a los factores de riesgo $\mathrm{y}$ consecuencias negativas de la violencia de pareja, de acuerdo con Rosales, Bedón, Díaz, Brioso y Pacheco (2016), la depresión funge como factor relacionado a la violencia. Según informa la OMS (2013), las mujeres que han sufrido violencia de pareja presentan casi el doble de probabilidades de presentarla.

Las consecuencias negativas relacionadas con la violencia de pareja pueden suceder en forma de cascada, es decir, que una consecuencia negativa afecte a otras (Cicchetti, 2013), así, dentro de los efectos negativos de la depresión se encuentra la afectación de la autorregulación emocional y conductual (Cuervo \& Izzedin, 2007). La autorregulación es un proceso psicológico que hace posible modificar las conductas con respecto a la demanda de una 
situación específica y permite mejorar el grado de adaptación del sujeto al medio, debido al manejo de las emociones negativas de una forma más adaptativa (Ibídem); las estrategias de autorregulación están relacionadas con diversos aspectos de la vida del ser humano, en especial con el rol de la crianza y disciplina de los hijos. Algunas madres que presentan problemas de autorregulación emocional y conductual tienen alta probabilidad de culminar con estrategias relacionadas con el maltrato físico y emocional de sus hijos (Doan, Fuller-Rowell, \& Evans, 2012). Por lo anterior, es importante también estudiar esta variable.

El maltrato infantil se define como los abusos y la desatención a la que son objeto los menores de 18 años, incluyendo maltrato físico o psicológico, abuso sexual, desatención, negligencia y explotación comercial o de otro tipo que causen o puedan causar un daño a la salud, desarrollo o dignidad del niño, o poner en peligro su supervivencia, en el contexto de una relación de responsabilidad, confianza o poder (OMS, 2013).

\section{Factores protectores de la violencia de pareja} hacia la mujer: apoyo social y bienestar psicológico

De acuerdo con la literatura, los factores protectores pueden modificar, mejorar o alterar la respuesta de un individuo ante algún peligro que lleva a un resultado no adaptativo (Bisquerra, 2008). El apoyo social percibido ha sido identificado como un factor protector para las mujeres que experimentan violencia de pareja (Suvak, Taft, Goodman, \& Dutton, 2013). Lin (1986) estructuró el concepto de apoyo social, planteándolo como provisiones expresivas que la comunidad, redes sociales y las personas establecen mediante vínculos de confianza, y se proporcionan a los individuos de la comunidad. A raíz de esta conceptualización han surgido diversas investigaciones referentes al apoyo social brindado a personas en situaciones difíciles o conflictivas.

De acuerdo con Hombrados y Castro (2013), el apoyo social incluye ayudas emocionales, instrumentales e informacionales. La ayuda emocional hace referencia al sentimiento personal por el hecho de ser amado y la seguridad de poder confiar en alguien; la ayuda instrumental alude a la posibilidad de disponer de ayuda de manera directa y con cosas tangibles, mientras que la ayuda informacional, se presenta como la aportación de una guía para resolver problemas. La ayuda que proviene de amigos, vecinos y familia, se conoce como apoyo social informal y se sustenta en el aprecio y la preocupación por el bienestar personal otorgado por los demás (ElBassel, Gilbert, Rajah, Folleno, \& Frye, 2001). El efecto positivo del apoyo social percibido en las mujeres víctimas de violencia de pareja puede establecerse a partir de que tiene el potencial de mejorar su bienestar psicológico (Vivaldi \& Barra, 2012; Lila, García, \& Murgui, 2013). 
El bienestar psicológico, según Fernández, García y Lorenzo (2014), es una dimensión subjetiva asociada a la calidad de vida, donde existe un juicio de valor con enfoque en la satisfacción con la vida, relacionado con las expectativas y logros obtenidos. Aquí entra en juego la resiliencia, la cual para Luthar (2000), resulta de la interacción entre persona y ambiente, donde para la conducta y el desarrollo es importante la percepción que se tenga del ambiente.

El bienestar psicológico ha sido señalado como factor protector de la violencia de pareja (Beeble, Bybee, Sullivan, \& Adams, 2009), de la depresión (Brown, 2000) y del maltrato infantil (Armstrong, Birnie-Lefcovitch, \& Ungar, 2005). A su vez, el nivel de bienestar psicológico se relaciona con la crianza autoritativa (Belsky, 1984) y las tendencias individuales para la superación de los riesgos (Morales \& González, 2014). Éstas últimas pueden ser denominadas disposiciones a la resiliencia y pueden afectar negativamente los problemas de autorregulación emocional y conductual (Gaxiola, González, Domínguez, \& Gaxiola, 2013), fungiendo como factor protector de dicha variable. Al mismo tiempo, las disposiciones a superar los riesgos pueden afectar positivamente la crianza autoritativa (Bradley, Davis, Wingo, Mercer, \& Ressler, 2013).

Para la teoría del desarrollo de la psicopatología, las mujeres que experimenten violencia de pareja no tendrán las mismas consecuencias negativas que pueden tener otras mujeres, debido a la interacción de los factores de riesgo con factores protectores psicológicos $\mathrm{y}$ sociales, lo cual posibilita una trayectoria que finalice con estilos de crianza autoritativa, caracterizada por altos niveles de control disciplinario con calidez y afecto (Baumrind, 1991), contrario al maltrato infantil. La crianza autoritativa se ha denominado también crianza positiva debido a sus efectos en el ajuste académico, social y personal de los hijos(as) (Healey, Flory, Miller, \& Halperin, 2011; Isaza \& Henao, 2012).

\section{Objetivo e hipótesis}

El objetivo del presente estudio fue evaluar los factores de riesgo de la violencia de pareja: depresión, problemas de autorregulación y maltrato infantil, así como los factores protectores: el apoyo social percibido de los amigos, familiares y vecinos, bienestar psicológico, disposiciones a la resiliencia, que hacen probable la crianza autoritativa.

La hipótesis plantea que el apoyo social percibido tiene efectos positivos en el bienestar psicológico. Además, el bienestar psicológico tiene efectos positivos sobre la crianza autoritativa $y$ efectos negativos sobre la violencia de pareja, la depresión, las disposiciones a la resiliencia y el maltrato infantil. Por otra parte, las disposiciones a la resiliencia afectan negativamente los problemas de autorregulación y positivamente la crianza autoritativa. El modelo hipotético se presenta en la figura 1. 


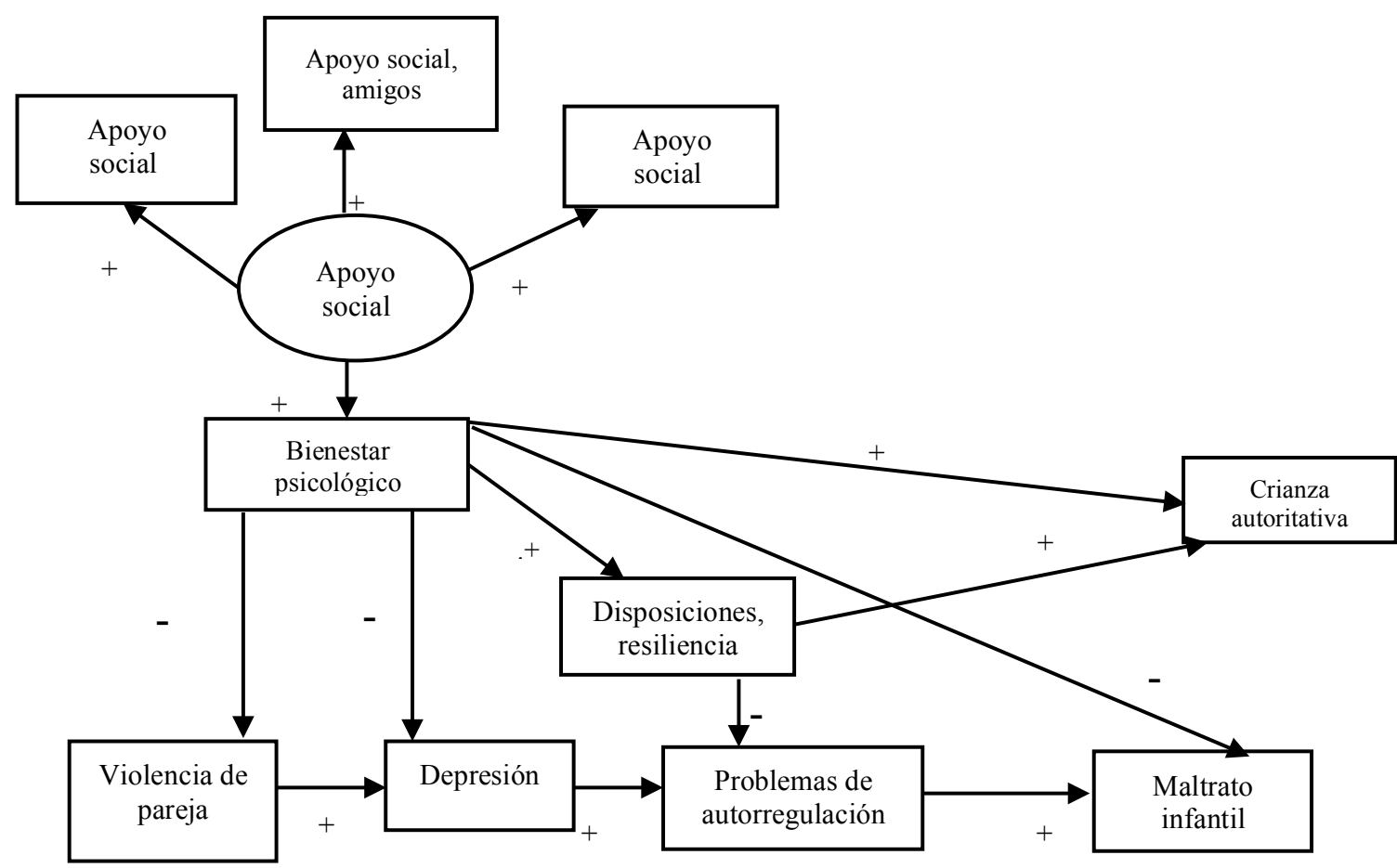

Figura 1. Modelo hipotético por probar sobre las relaciones de factores protectores y de riesgo de la crianza materna en contexto de violencia de pareja.

Nota: los signos representan el tipo de relación esperada.

\section{Método}

El diseño que utilizamos fue de tipo transversal, no probabilístico y de alcance correlacional; se utilizó la herramienta estadística de ecuaciones estructurales (Bentler, 2006).

\section{Participantes}

Fue seleccionado un total de 254 encuestas de madres con al menos una experiencia de violencia física, emocional o sexual en una relación de pareja. Las madres fueron contactadas por medio de las escuelas donde estudiaban sus hijos. Se seleccionaron 20 escuelas, 10 de ellas con alto logro académico y 10 con bajo logro, según el reporte de la prueba ENLACE 2011 (SEP, 2012). Otro de los criterios de inclusión fue que las madres vivieran con su pareja heterosexual en el momento de la entrevista, puesto que la variable de violencia de pareja en las estadísticas (INEGI, 2016) se presenta en cuestión de parejas heterosexuales. En la tabla 1 se muestran los datos demográficos.

\section{Instrumentos}

Se construyó una batería de pruebas que preguntaba, en principio, datos demográficos como edad de la madre, número de hijos(as), ingreso mensual de las madres y el ingreso familiar. 
Cada una de las escalas fue validada previamente en una población similar a la del presente estudio. Las características de los instrumentos se pueden ver en la siguiente tabla 2.

\section{Tabla 1}

Estadísticas descriptivas de las madres de la muestra

\begin{tabular}{lccccc}
\hline \multicolumn{1}{c}{ Variables } & $\mathrm{N}$ & Media & DT & Mínimo & Máximo \\
\hline Edad de madres & 245 & 35.24 & 5.8 & 25 & 58 \\
Número de hijos(as) & 253 & 2.99 & 1.1 & 1 & 6 \\
Ingreso mensual madres & 243 & 95.06 & 195.38 & 0 & 2114.8 \\
Ingreso familiar & 243 & 376.87 & 416.47 & 0 & 3625.37 \\
\hline
\end{tabular}

Nota. DT = desviación típica; Ingreso familiar = el ingreso familiar que originalmente las participantes señalaron en pesos mexicanos, se convirtió a dólares estadounidenses.

\section{Tabla 2}

\section{Descripción de instrumentos utilizados en el estudio}

\begin{tabular}{|c|c|c|c|c|}
\hline Variable & Tipo de escala & No. de ítems & Ejemplo de ítem & Alpha \\
\hline $\begin{array}{l}\text { Estilos de } \\
\text { crianza autori- } \\
\text { tativa }\end{array}$ & $\begin{array}{l}\text { Escala hecha exprofeso para el estudio, } \\
\text { que evaluó las dimensiones afectivas y de } \\
\text { control de los hijos, basada en la definición } \\
\text { de Baumrind }(1991) \text {. Tipo Likert que va de } \\
0=\text { nunca y } 4=\text { siempre }\end{array}$ & 11 ítems & $\begin{array}{l}\text { "Le dice las cosas que } \\
\text { hace bien" }\end{array}$ & .74 \\
\hline $\begin{array}{l}\text { Autor- } \\
\text { regulación } \\
\text { conductual y } \\
\text { emocional }\end{array}$ & $\begin{array}{l}\text { Versión breve traducida al español, de } \\
\text { Behavior Rating Inventory of Executive } \\
\text { Functions (BRIEF) de Gioia, Isquith, } \\
\text { Retzlaff y Espy (2002). Tipo Likert desde } 0 \\
=\text { nunca a } 6=\text { siempre }\end{array}$ & 30 ítems & $\begin{array}{l}\text { "Tiene explosiones de } \\
\text { enojo" }\end{array}$ & .90 \\
\hline Depresión & $\begin{array}{l}\text { Depresión de Hamilton (Hamilton, \& } \\
\text { Collins, 1981). Tipo Likert } 0=0 \text { días }-14 \\
=14 \text { días. }\end{array}$ & 10 ítems & $\begin{array}{l}\text { "Indique en las dos } \\
\text { últimas semanas: } \\
\text { ¿cuántos días se ha } \\
\text { sentido mal por haber } \\
\text { fallado en algo? }\end{array}$ & .91 \\
\hline $\begin{array}{l}\text { Violencia de } \\
\text { pareja }\end{array}$ & $\begin{array}{l}\text { Adaptación de la traducción de la escala } \\
\text { de Tácticas de Conflicto de Straus (1990). } \\
\text { Tipo Likert de } 0=\text { nunca, y } 6=\text { más de } \\
\text { veinte veces }\end{array}$ & 22 ítems & $\begin{array}{l}\text { ¿Cuántas veces su } \\
\text { pareja la ha insul- } \\
\text { tado o dicho malas } \\
\text { palabras?” }\end{array}$ & .84 \\
\hline
\end{tabular}


Tabla 2

Continuación

\begin{tabular}{|c|c|c|c|c|}
\hline Variable & Tipo de escala & No. de ítem & Ejemplo de ítem & Alpha \\
\hline $\begin{array}{l}\text { Percepción de } \\
\text { apoyo social } \\
\text { de los amigos } \\
\text { y familiares }\end{array}$ & $\begin{array}{l}\text { Traducción al español del inventario DICA- } \\
\text { R-7.3 (Reich, 1992). } \\
\text { Escala tipo Likert, con opciones de respues- } \\
\text { ta de 0-6 }\end{array}$ & 10 ítems & $\begin{array}{l}\text { ¿Con qué frecuencia } \\
\text { un amigo demostró } \\
\text { interés y preocupación } \\
\text { por su bienestar? } \\
\text { ¿Con qué frecuencia } \\
\text { algún familiar le dio } \\
\text { dinero?” }\end{array}$ & .92 \\
\hline $\begin{array}{l}\text { Percepción del } \\
\text { apoyo social } \\
\text { de los vecinos }\end{array}$ & $\begin{array}{l}\text { Traducción al español de la escala de Se- } \\
\text { guridad Comunitaria/Apoyo Comunitario } \\
\text { (Community Safety/Support) contenidas en } \\
\text { el instrumento Trauma Resilience Scale for } \\
\text { Children "TRS-C" (Thompson, 2010). Tipo } \\
\text { likert de } 0 \text { a } 4\end{array}$ & 9 ítems & $\begin{array}{l}\text { "Mis vecinos me } \\
\text { apoyan" }\end{array}$ & .84 \\
\hline $\begin{array}{l}\text { El bienestar } \\
\text { psicológico }\end{array}$ & $\begin{array}{l}\text { Inventario desarrollado para el estudio at- } \\
\text { endiendo algunos indicadores, los cuales } \\
\text { fueron autonomía, relaciones positivas, } \\
\text { propósito en la vida, autoaceptación, do- } \\
\text { minio del entorno y crecimiento personal } \\
\text { (Ryff, 1989). Tipo Likert de } 1=\text { nada, a } 5 \\
=\text { totalmente }\end{array}$ & 12 ítems & $\begin{array}{l}\text { "Se siente capaz de re- } \\
\text { solver sus problemas" }\end{array}$ & .88 \\
\hline $\begin{array}{l}\text { Las disposi- } \\
\text { ciones a la } \\
\text { resiliencia }\end{array}$ & $\begin{array}{l}\text { Inventario de Resiliencia (IRES) tipo Lik- } \\
\text { ert (Gaxiola, Frías, Hurtado, Salcido, \& } \\
\text { Figueroa, 2011). } 0=\text { nada a } 5=\text { siempre. }\end{array}$ & 20 ítems & $\begin{array}{l}\text { "En el último mes } \\
\text { fue capaz de sonreír a } \\
\text { pesar de sus proble- } \\
\text { mas" }\end{array}$ & .93 \\
\hline
\end{tabular}

\section{Procedimiento}

Primeramente, se eligieron las escuelas, 10 escuelas de alto logro académico y 10 escuelas de bajo logro, según el reporte de la prueba ENLACE 2011 (SEP, 2012). Más tarde, se contactó a los directivos de cada una de las escuelas para explicarles el estudio, con el fin de obtener los permisos correspondientes y así poder contactar a las madres de los niños(as) de $5^{\circ}$ y $6^{\circ}$ grado de primaria. Fue solicitada de manera escrita la participación voluntaria de las madres para responder la encuesta que sus hijos les llevaban en un sobre cerrado, mismo que los niños debían regresar al día siguiente contestado por su madre.

\section{Análisis de datos}

Los datos fueron capturados en el programa estadístico SPSS 21. Se realizaron 
las estadísticas descriptivas de las variables demográficas. Asimismo, se obtuvieron índices de las escalas, que fueron correlacionados con el objetivo de analizar la pertinencia general del modelo hipotético propuesto. Para el caso del apoyo social, se agruparon las escalas con base en tres factores en un analisis factorial confirmatorio con el fin de agruparlos en una sola escala y correlacionarlos de manera conjunta. Posteriormente, se emplearon ecuaciones estructurales para probar el modelo hipotético utilizando el paquete estadístico EQS 6.1. Los indicadores de bondad de ajuste utilizados fueron el valor de chi cuadrada $\left(X^{2}\right)$, la cual se esperaba que resultara no significativa, o bien, que tenga una relación con los grados de libertad $\left(X^{2} / g l\right)$ menor a dos para aceptarse el modelo; además, se requiere que los valores de los indicadores de bondad de ajuste prácticos BBNFI y el CFI, presenten valores superiores a .90, así como un valor RMSEA cercano a cero, con valores menores a .08 (Bentler, 2006; Hu \& Bentler, 1999).

\section{Resultados}

Los coeficientes de alpha de Cronbach obtenidos para cada una de las escalas de medida se encuentran en la tabla 3. Cabe mencionar que todos los valores de alpha fueron $\geq .60$, por lo cual fueron considerados valores aceptables (Nieva \& Sorra, 2003).

Tabla 3

Valores de Alpha de Cronbach de las escalas usadas en la investigación

\begin{tabular}{ll}
\hline Variables & Alpha de Cronbach \\
\hline & .93 \\
Apoyo social amigos & .94 \\
Apoyo social familia & .86 \\
Apoyo social vecinos & .80 \\
Bienestar psicológico & .93 \\
Disposiciones resiliencia & .94 \\
Violencia de pareja & .93 \\
Depresión & .84 \\
Problemas autorregulación & .78 \\
Crianza autoritativa & \\
\hline
\end{tabular}


En la tabla 4 se muestran las correlaciones y se puede observar que en su mayoría las variables del modelo hipotético se correlacionaron entre sí de manera significativa $(p \leq .05, p \leq .01)$, tanto positiva como negativamente, según lo propuesto. Las únicas que no resultaron significativas fueron las correlaciones entre depresión $(\mathrm{r}=-.03)$, autorregulación $(\mathrm{r}=-.09)$, y maltrato infantil $(r=-.10)$, con el apoyo social percibido.

\section{Tabla 4}

Correlaciones de Pearson de las variables de la investigación

\begin{tabular}{lllllllll}
\hline Variable & Apoyos & Bien & Resil & Crianza & Violen & Depre & Auto & Mal \\
\hline Apoyos & 1 & & & & & & & \\
Bien & $.22^{* *}$ & 1 & & & & & & \\
Resil & $.21^{* *}$ & $.74^{* *}$ & 1 & & & & & \\
Crianza & $.16^{*}$ & $.47^{* *}$ & $.46^{* *}$ & 1 & & & & \\
Violen & $-.12^{*}$ & $-.42^{* *}$ & $.31^{* *}$ & $-.18^{* *}$ & 1 & & & \\
Depre & -.03 & $-.53^{* *}$ & $-.39^{* *}$ & $-.34^{* *}$ & $.49^{* *}$ & 1 & & \\
Auto & -.09 & $-.35^{* *}$ & $-.33^{* *}$ & $-.30^{* *}$ & $.28^{* *}$ & $.55^{* *}$ & 1 & \\
Mal & -.10 & $-.28^{* *}$ & $-.20^{* *}$ & $-.25^{* *}$ & $.43^{* *}$ & $.35^{* *}$ & $.32^{* *}$ & 1 \\
\hline
\end{tabular}

${ }^{*} p \leq .05,{ }^{* *} p \leq .01, \mathrm{~N}=254$

Nota . Apoyos $=$ Apoyos sociales, Bien $=$ Bienestar psicológico, Resil $=$ Disposiciones a la resiliencia, Crianza $=$ Crianza autoritativa, Violen $=$ Violencia de pareja, Depre $=$ depresión, Auto $=$ Problemas de autorregulación emocional $\mathrm{y}$ conductual, $\mathrm{Mal}=$ maltrato infantil).

A partir de la literatura se propuso un modelo teórico (figura 1) que fue contrastado con el modelo empírico obtenido con los datos (figura 2 ), en donde se incluyeron todas las variables del estudio. En el caso del factor Apoyo social, se conformó con los tres tipos de apoyos sociales evaluados, el cual presentó efectos en el modelo a partir del bienestar psicológico. El indicador de bondad de ajuste práctico (CFI), fue superior a .90 , sin embargo, el BNFI fue de .86, lo cual implica que el modelo puede mejorarse para estudios posteriores, incluyendo mayor número de ítems o aumentando el tamaño de la muestra. Por otra parte, se obtuvo una Chi cuadrada significativa de $\mathrm{X}^{2}=98.28$ (gl $=70) \mathrm{p}=0.0001$, lo cual indica que el modelo sintético fue diferente del modelo resultante, por lo tanto, para determinar la pertinencia del modelo propuesto, se utilizó la relación entre el valor de $\mathrm{X}^{2}$ y los grados de libertad menor a 3 
(Ruiz, Pardo, \& San Martín, 2010). El valor de $\mathrm{X}^{2}$ entre los grados de libertad fue $\leq 3\left(\mathrm{X}^{2} / \mathrm{gl}\right)$ $98.28 / 70=1.9$, por ende, en términos generales, el modelo teórico se ajustó a los datos (Bentler, 2006) y explica $25 \%$ de la crianza autoritativa y el $13 \%$ del maltrato infantil.

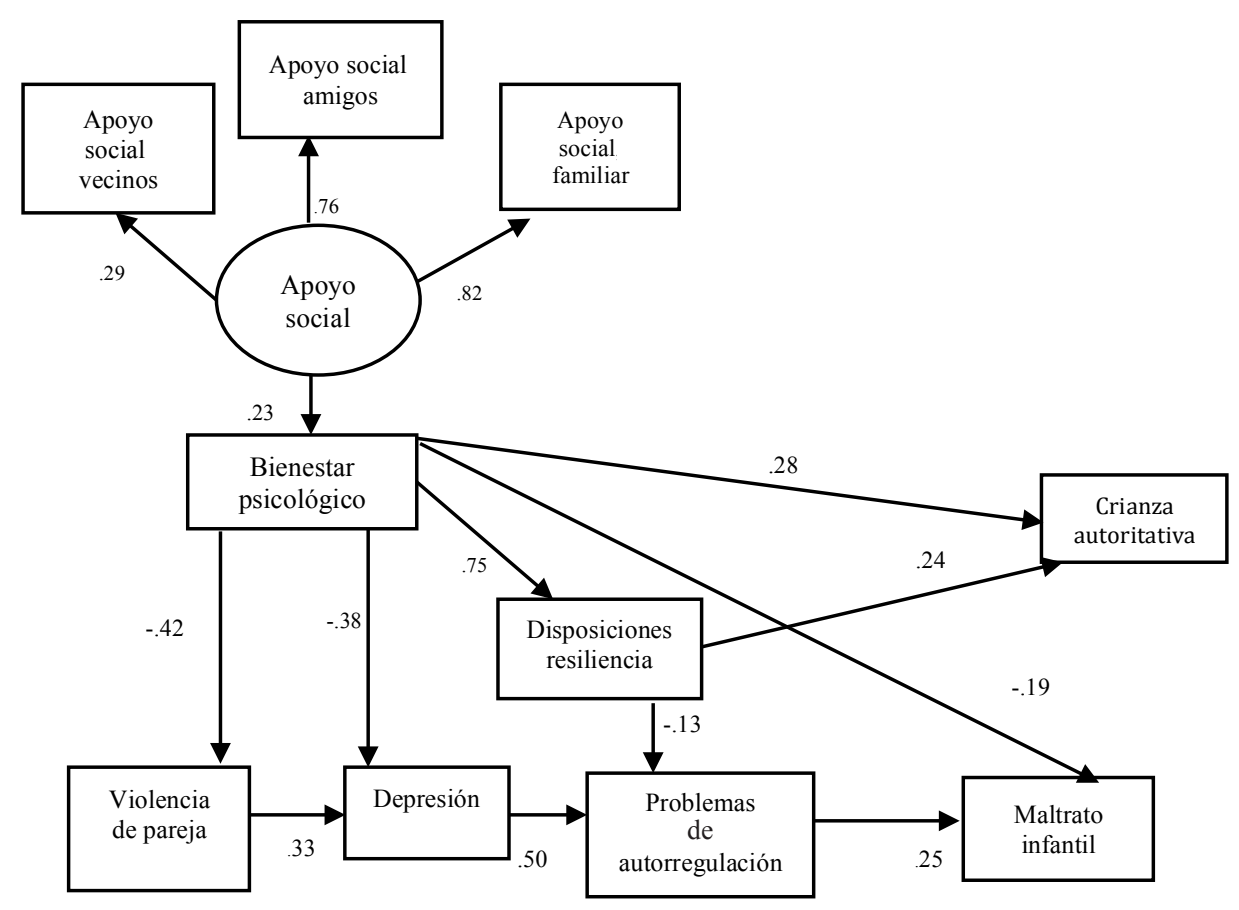

$\mathrm{X}^{2}=98.28(70 \mathrm{gl}), \mathrm{p}<.0001 ; \mathrm{BBNFI}=.88, \mathrm{CFI}=.91, \mathrm{RMSEA}=.09(90 \% \mathrm{IC}=.07-.11) . \mathrm{R}^{2}$ Crianza autoritativa $=.25 ; \mathrm{R}^{2}$ Maltrato infantil $=.13$.

Figura 2. Modelo sobre sobre factores protectores y de riesgo de la crianza materna en contexto de violencia de pareja.

\section{Discusión}

El objetivo de la investigación fue evaluar los factores de riego y protectores de la violencia de pareja respecto a la crianza materna. El modelo que resultó mostró que la crianza autoritativa es posible, es decir, algunas mujeres en un contexto de violencia de pareja sí encuentran los apoyos sociales pertinentes, provenientes de los vecinos, de los amigos y del entorno familiar. El factor de apoyo social percibido tuvo gran peso respecto al bienestar psicológico, de ahí la importancia para algunas madres que padecen violencia de pareja. Sin embargo, parece sencillo que las mujeres con violencia de pareja obtengan el apoyo social de diversas personas $y$, por ende, presenten al menos un poco de bienestar psicológico, pero no 
olvidemos que una característica de las mujeres en tal situación es precisamente su aislamiento social (Katerndahl, Burge, Ferrer, Becho, \& Wood, 2013).

De acuerdo con los resultados, el nivel de bienestar psicológico fue afectado directamente por el apoyo social, lo cual ha sido señalado por investigaciones desarrolladas en diversos lugares del mundo (Vivaldi \& Barra, 2012; Cachimuel \& Granda, 2013). Por lo tanto, entre mayor es el sistema de apoyo social de las mujeres víctimas de violencia, existe mayor probabilidad de presentar cierto nivel de bienestar psicológico. Una manera de fomentar el apoyo social en las mujeres en riesgo de sufrir violencia de pareja es aprovechar los grupos sociales existentes en la comunidad y organizar actividades compartidas entre mujeres, de este modo, dar pie a la cercanía y confianza entre ellas, elemento base para el desarrollo de los apoyos sociales.

El modelo indicó también que el bienestar psicológico se relaciona negativamente con la violencia de pareja, con la depresión y el maltrato infantil, por lo cual puede interpretarse como un factor de protección, relacionado con el apoyo social. Investigaciones han señalado que aquellas mujeres con bienestar psicológico permitirán menos la presencia de actos agresivos de sus parejas (Anderson \& Saunders, 2003), asimismo, es posible que las mujeres al tener apoyo social permanezcan menos tiempo en casa y, entonces, tengan menos probabilidad de ser agredidas. Se ha señalado que el estado emocional producto del bienestar psicológico es contrario a la depresión experimentada por las víctimas de violencia de pareja; mayores niveles de bienestar psicológico afectarán la sintomatología de la depresión, como lo corroboran Beeble y colaboradores (2009).

Por su parte, el bienestar psicológico de las madres se relaciona negativamente con los reportes de maltrato infantil como refieren Armstrong y compañeros (2005), y de manera positiva con el estilo de crianza autoritativo, como también lo describen Simons y Johnson (1996). Se requieren ciertos niveles de bienestar psicológico para poder mantener estilos de crianza caracterizados por el control disciplinario y el intercambio afectivo, mientras se supervisa y disciplina el comportamiento de los hijos(as). De manera similar a lo que se encontró en el presente estudio, el control y estabilidad de la crianza se han asociado con el bienestar psicológico de las madres (Laukkanen, Ojansuu, Tolvanen, Alatupa, \& Aunola, 2014).

Al abordar la parte superior del modelo hipotético, donde están los factores protectores, se puede observar el bienestar psicológico, relacionado positivamente con las disposiciones a la resiliencia, por lo tanto, es probable que se presenten dimensiones personales asociadas con la superación de los riesgos. De acuerdo con la propuesta de medición de resiliencia utilizada en la presente investigación, las disposiciones se deben relacionar con una competencia de acuerdo con la edad y la cultura. En este caso, la resiliencia obtuvo una relación directa y positiva con la crianza autoritativa, como se ha indicado 
en otras investigaciones (Schofield, Conger, \& Neppl, 2014).

En la parte inferior del modelo, se muestra una trayectoria de los factores de riesgo de la violencia de pareja, donde ésta se relaciona con la depresión, igual que se ha confirmado en otras investigaciones (OMS, 2013). Además, la depresión se relacionó positivamente con los problemas de autorregulación, como lo apoyan otros estudios (Cuervo \& Izzedin, 2007) y, por último, tal como en otras investigaciones (Siegel, 2013), los problemas de autorregulación afectaron al maltrato infantil.

Ante el panorama mostrado por los datos, son posibles dos trayectorias, una negativa $\mathrm{y}$ otra positiva en el contexto de violencia de pareja, como lo indica la teoría del desarrollo de la psicopatología (Cicchetti, 2006). Dependerá de los efectos positivos de los apoyos sociales disponibles, que pueda culminar una en la trayectoria en estilos de crianza autoritativa, para evitar la transmisión de la violencia de pareja con los hijos. Es necesario tomar en cuenta que se requieren de estudios longitudinales para comprobar la direccionalidad de las relaciones de las variables presentes en esta investigación.

El modelo tiene una varianza explicada de $25 \%$ con la crianza autoritativa y sólo $13 \%$ de explicación del modelo respecto al maltrato infantil, en consecuencia, es imprescindible considerar otras variables en la explicación del fenómeno de la crianza de los hijos y el maltrato infantil, por ejemplo, las habilidades de crianza retroalimentadas por los apoyos sociales, proporcionadas por el nivel educativo de las madres (Ellingsen, Baker, Blacher, \& Crnic, 2014).

La investigación presenta varias limitaciones, entre ellas, el hecho de que la muestra fue no probabilística y las encuestas fueron autoadministradas, razón por la cual puede existir un sesgo de deseabilidad social en los resultados, además, se debe tomar en cuenta que las respuestas están limitadas a la percepción de las madres sobre cada uno de los tópicos evaluados y no se realizó otro tipo de evaluaciones para corroborar lo que ellas respondían. Otra limitación del estudio es que no se hicieron análisis diferenciales sobre la temporalidad, frecuencia e intensidad de la violencia experimentada por las madres.

Los indicadores del modelo resultantes no fueron perfectamente ajustados, por consiguiente, la presente investigación puede tomarse como una exploración, ya que indica la posible relación entre las variables implicadas, pero requiere de réplicas en otras poblaciones considerando las limitaciones del presente estudio. A pesar de lo anterior, los datos pueden considerarse como base para el estudio de las limitaciones en otros lugares con mujeres en riesgo de padecer violencia de pareja, para evitar la reproducción de la violencia que reciben las mujeres de sus parejas, en forma de maltrato infantil con sus propios hijos. 


\section{Referencias}

Alonso, M., Manso, J., García-Baamonde, \& Sánchez, M. (2009). Inteligencia emocional como alternativa para la prevención del maltrato psicológico en la pareja. Anales de Psicología, 2(25), 250-260. doi: $10.6018 / 87541$

Anderson, D. K., \& Saunders, D. G. (2003). Leaving an abusive partner an empirical review of predictors, the process of leaving, and psychological well-being. Trauma, Violence, \& Abuse, 4(2), 163-191. doi: 10.1177/1524838002250769

Armstrong, M. I., Birnie-Lefcovitch, S., \& Ungar, M. T. (2005). Pathways between social support, family wel- being, quality of parenting, and child resilience: What we know. Journal of Child and Family Studies, 14(2), 269-281. doi: 10.1007/s10826-0055054-4

Baumrind, D. (1991). The influence of parenting style on adolescent competence and substance use. Journal of Early Adolescence, 11(1), 56-95. Recuperado de http://mltei. org/cqn/Adolescent\%20Development/ Resources/Family/Baumrind,\%20The\%20 influence $\% 20$ of $\% 20$ parenting $\% 20$ style $\% 20$ on $\% 20$ adolescent $\% 20$ competence $\% 20$ and $\% 20$ substance $\% 20$ use.pdf

Beeble, M. L., Bybee, D., Sullivan, C. M., \& Adams, A. E. (2009). Main, mediating, and moderating effects of social support on the well-being of survivors of intimate partner violence across 2 years. Journal of consulting and clinical psychology, 77(4), 718-729. doi: 10.1037/a0016140

Belsky, J. (1984). The determinants of parenting: A process model. Child development, 55(1), 83-96. doi: 10.2307/1129836

Bentler,P.M.(2006). EQS 6 StructuralEquations Program Manual. CA: Mulivariate Software Inc.

Bisquerra, R. (2008). Educación para la ciudadanía y convivencia: El enfoque de la Convivencia Emocional. España: Wolters Kluwer/Universidad Nacional de Educación a Distancia España.

Bradley, B., Davis, T. A., Wingo, A. P., Mercer, K. B., \& Ressler, K. J. (2013). Family environment and adult resilience: contributions of positive parenting and the oxytocin receptor gene. European Journal of Psychotraumatology, 4(1), 21659. Doi:10.3402/ejpt.v4i0.21659

Brown, S. L. (2000). The effect of union type on psychological well-being: Depression among cohabitors versus marrieds. Journal of health and social behavior. 41(3), 241-255. doi: 10.2307/2676319

Buesa, S., \& Calvete, E. (2013). Violencia contra la mujer y síntomas de depresión y estrés postraumático: el papel del apoyo social. International Journal of Psychology and Psychological Therapy, 13(1) 31-45.

Cachimuel, M., \& Granda, M. (2013). Violencia intrafamiliar, aplicabilidad de la Ley 103 en mujeres atendidas en el Ministerio Público de la ciudad de Ibarra período de enero 
a diciembre del 2006. (Tesis inédita de Licenciatura). Ecuador: Universidad Técnica del Norte.

Cicchetti, D. (2006). Development and psychopathology. En D. Cicchetti \& D. J. Cohen (Eds.), Developmental Psychopathology: Risk, Disorder, and Adaptation, 2nd edition (pp. 1-23). New York: Wiley.

Cicchetti, D. (2013). Annual research review: Resilient functioning in maltreated childrenpast, present, and future perspectives. Journal of child psychology and psychiatry, 54(4), 402-422.

Cuervo, M. A., \& Izzedin, B. R. (2007). Tristeza, depresión y estrategias de autorregulación en niños. Tesis Psicológica, 1(2), 35-47. Recuperado de http://www.redalyc.org/ articulo.oa?id=139012670004

Darling, N., \& Steinberg, L. (1993). Parenting style as a context: An integrative model. Psychological, 113, 487-496.

Doan, S. N., Fuller-Rowell, T. E., \& Evans, G. W. (2012). Cumulative risk and adolescent's internalizing and externalizing problems: The mediating roles of maternal responsiveness and self-regulation. Developmental Psychology, 48(6), 1529-1539. doi: 10.1037/ a0027815

El-Bassel, N., Gilbert, L., Rajah, V., Folleno, A., \& Frye, V. (2001). Social support among women in methadone treatment who experience partner violence: Isolation and male controlling behavior. Violence Against Women, 7(3), 246-275. doi: $10.1177 / 10778010122182433$

Ellingsen, R., Baker, B. L., Blacher, J., \& Crnic, K. (2014). Resilient parenting of children at developmental risk across middle childhood. Research in Developmental Disabilities, 35(6), 1364-1374. doi: 10.1016/j. ridd.2014.03.016

Fernández, A., García, C., \& Lorenzo, A. (2014). Consideraciones acerca del bienestar psicológico. Revista Electrónica de Psicología Iztacala, 17(3), 1108-1146.

Frías, M. (2002). Long-term effects of child punishment on Mexican women: A structural model. Child Abuse and Neglect, 4(26), 371386. doi: 10.1016/S0145-2134(02)00314-9

Gaxiola, J. C., Frías, M., Hurtado, M. F., Salcido, L. C., \& Figueroa, F. M. (2011). Validación del inventario de resiliencia (IRES) en una población del noroeste de México. Enseñanza e Investigación en Psicología, 1(16), 7383. Recuperado de http://www.redalyc.org/ articulo.oa? $\mathrm{id}=29215963006$

Gaxiola, J. C., González, S., Domínguez, M., \& Gaxiola, E. (2013). Autorregulación, metas $\mathrm{y}$ rendimiento académico en bachilleres con disposiciones resilientes y no resilientes. Revista Interamericana de Psicología, 47(1), 71-81. Recuperado de http://www.redalyc. org/articulo.oa?id=28426980009

Gioia, G. A., Isquith, P. K., Retzlaff, P. D., \& Espy, K. A. (2002). Confirmatory factor 
analysis of the Behavior Rating Inventory of Executive Function (BRIEF) in a clinical sample. Child Neuropsychology, 8(4), 249257. Doi: 10.1076/chin.8.4.249.13513

Hamilton, C. J., \& Collins, J. J. (1981). The role of alcohol in wife beating and child abuse: A review of the literature. En J. J. Collins (Ed.), Drinking and Crime: Perspectives on the Relationships between Alcohol Consumption and Criminal Behavior (pp. 253-287). New York: Guilford Press.

Healey, D. M., Flory, J. D., Miller, C. J., \& Halperin, J. M. (2011). Maternal positive parenting style is associated with better functioning in hyperactive/inattentive preschool children. Infant and Child Development, 20(2), 148-161. doi: 10.1002/ icd.682

Hombrados-Mendieta, I., \& CastroTravé, M. (2013). Apoyo social, clima social y percepción de conflictos en un contexto educativo intercultural. Anales de Psicología, 29(1), 108-122. Recuperado de http://scielo.isciii.es/ scielo.php? script $=$ sci_arttext\&pid $=$ S021297282013000100014\&lng=es.http://dx.doi. org/10.6018/analesps.29.1.123311.

Hu, L., \& Bentler, P. M. (1999). Cutoff criteria for fit indexes in covariance structure analysis: conventional criteria versus new alternatives. Structural Equation Modeling, 6(1), 1-55. doi: 10.1080/10705519909540118

Instituto Nacional de Estadística, Geografía. (2016). Estadísticas a propósito del día internacional de la eliminación de la violencia contra la mujer (25 de noviembre). Recuperado de http://www. inegi.org.mx/saladeprensa/aproposito/2016/ violencia2016_0.pdf

Isaza, L., \& Henao, G. C. (2012). Influencia del clima sociofamiliar y estilos de interacción parental sobre el desarrollo de habilidades sociales en niños y niñas. Persona, 15 (enerodiciembre), 253-271. Recuperado de http:// revistas.ulima.edu.pe/index.php/Persona/ article/view/138

Jose, R., \& Novaco, R. W. (2016). Intimate Partner Violence Victims Seeking a Temporary Restraining Order: Social Support and Resilience Attenuating Psychological Distress. Journal of interpersonal violence, 31(20), 3352-3376.

Katerndahl, D., Burge, S., Ferrer, R., Becho, J., \& Wood, R. (2013). Differences in social network structure and support among women in violent relationships. Journal of Interpersonal Violence, 28(9), 1948-1964. doi: $10.1177 / 0886260512469103$

Laukkanen, J., Ojansuu, U., Tolvanen, A., Alatupa, S., \& Aunola, K. (2014). Child's difficult temperament and mothers' parenting styles. Journal of Child and Family Studies, 23(2), 312-323. doi: 10.1007/s10826-0139747-9

Lila, M., García, E., \& Murgui, S. (2013). Psychological adjustment and victimblaming among intimate partner violence offenders: The role of social support and 
stressful life events. The European Journal of Psychology Applied to Legal Context, 5, 147-153. doi:10.5093/ejpalc2013a4

Limiñana, A. R., Martínez, R. S., \& Pérez, M. A. M. (2017). Problemas de conducta infantil y competencias parentales en madres en contextos de violencia de género. 31 (3) Gaceta Sanitaria, Recuperado de https://doi. org/10.1016/j.gaceta.2017.02.004

Lin, N. (1986). Conceptualizing social support. En N. Lin, A. Dean \& W. Ensel (Eds.), Social support, life events, and deppression (17-30). New York: Academic Press.

Luthar, S., Cicchetti, D., \& Becker, B. (2000). The Construct of resilience: $A$ critical evaluation and guidelines for future work, Child Development, 71(3), 543-558.

Morales, M., \& González, A. (2014). ResilienciaAutoestima-Bienestar psicológico y Capacidad intelectual de estudiantes de cuarto medio de buen rendimiento de liceos vulnerables. Estudios Pedagógicos (Valdivia), 40(1), 215-228. doi: 10.4067/ S0718-07052014000100013

Nieva, V.F., \& Sorra, J. (2003). Safety culture assessment: a tool for improving patient safety in healthcare organizations. Quality Safe Health Care, 12 (supl), ii17-ii23.

Organización Mundial de la Salud. (2013a). Estimaciones mundiales y regionales de la violenciacontra la mujer:prevalenciay efectos de la violencia conyugal y de la violencia sexual no conyugal en la salud. WHO/RHR/
HRP/13.06. Recuperado de http://apps.who. int/iris/bitstream/10665/85243/1/WHO_ RHR_HRP_13.06_spa.pdf?ua $=1$

Organización Mundial de la Salud. (2013b). Organización Mundial de la Salud Violencia contra la mujer: Violencia de pareja y violencia sexual contra la mujer. Nota descriptiva N. ${ }^{\circ}$ 239. Recuperado de http:// www.who.int/mediacentre/factsheets/fs239/ es/

Organización Mundial de la Salud. (2014). Organización Mundial de la Salud Maltrato Infantil. Nota descriptiva $\mathrm{N} .^{\circ}$ 150. Recuperado de http://www.who.int/ mediacentre/factsheets/fs150/es/

Reich, W. (1992). Diagnostic Interview for Children and Adolescents-Revised (DICA-R-7.3). Washington: University School of Medicine/Division of Child Psychiatry.

Rosales, R. H., Bedón, M. B., Díaz, C. P., Brioso, A. S., \& Pacheco, E. C. (2016). Depresión $\mathrm{y}$ ansiedad en mujeres en situación de maltrato en la relación de pareja según tipo de convivencia, en zonas urbano-marginales de la ciudad de Lima. Theorēma (Lima, Segunda Época, En línea), (1), 123-136.

Ruiz, M. A., Pardo, A., \& San Martín, R. (2010). Modelos de ecuaciones estructurales. Papeles del psicólogo, 31(1).

Ryff, C. D. (1989). Happiness is everything, or is it? Explorations on the meaning of psychological well-being. Journal 
of Personality and Social Psychology, 6(57), 1069-1081. doi: http://dx.doi. org/10.1037/0022-3514.57.6.1069

Schofield, T. J., Conger, R. D., \& Neppl, T. K. (2014). Positive parenting, beliefs about parental efficacy, and active coping: Three sources of intergenerational resilience. Journal of Family Psychology, 28(6), 973978. doi: 10.1037/fam0000024

Secretaría de Educación Pública. (2012). Prueba Enlace 2011. Recuperado de http:// www.enlace.sep.gob.mx/ba/prueba_en_ linea_2011/

Siegel, J. P. (2013). Breaking the links in intergenerational violence: An emotional regulation perspective. Family Process, 52(2), 163-178. doi: 10.1111/famp.12023

Simons, R. L., \& Johnson, C. (1996). Mother's parenting. In R. L. Simons, Understanding families, Vol. 5. Understanding differences between divorced and intact families: Stress, interaction, and child outcome (pp. 81-93). Thousand Oaks, CA: Sage Publications.

Straus, M. A. (1990). The Conflict Tactics Scale and its critics: An evaluation and new data on validity and reliability. En M. A. Straus \& R. J. Gelles (Eds.), Physical Violence in American Families: Risk factors and adaptations to violence in 8,145 families. $\mathrm{NJ}$ : Transactions Publishers.

Suvak, M. K., Taft, C. T., Goodman, L. A., \& Dutton, M. A. (2013). Dimensions of functional social support and depressive symptoms: A longitudinal investigation of women seeking help for intimate partner violence. Journal of Consulting and Clinical Psychology, 81(3), 455-466. doi: 10.1037/ a0031787

Thompson, M. D. M. (2010). Trauma resilience scale for children: Validation of protective factors associated with positive adaptation following violence. (Doctoral thesis of Social Work). Recuperado de http://etd. lib.fsu.edu/theses/available/etd-11082010 115432 /unrestricted/Thompson_M_ Dissertation_2010.pdf

Vivaldi, F., \& Barra, E. (2012). Bienestar Psicológico, Apoyo social percibido y percepción de salud en adultos mayores. Terapia Psicológica, 2(30), 23-29. doi: 10.4067/S0718-48082012000200002

Recibido el 26 de agosto de 2017 Revisado el 07 de octubre de 2017 Aceptado el 14 de octubre de 2017 